\title{
Organiser's Report on the MeCCSA-PGN Conference 2-3 July 2015
}

\author{
FRANCIEN BROEKHUIZEN, POPPY WILDE, DANAI MIKELLI, Coventry University
}

\begin{abstract}
In this visual essay, the organising committee look back on the MeCCSA-PGN Conference 2015 and reflect on the overarching theme, the organisation and the running of the conference. The conference's theme, 'Transformative Practice and Theory: Where We Stand Today', forms the basis of this special issue of Networking Knowledge, with developed papers from the conference, as well as interviews with some of the keynote speakers, included in this issue.
\end{abstract}

\section{KEYWORDS}

Conference Organisation; MeCCSA-PGN; Postgraduate Events; Theory; Transformative Practice

\section{Introduction}

The MeCCSA-PGN Conference 2015 took place at Coventry University on the 2-3 July, with a pre-conference screening of the documentary CitizenFour (2014) the day before to enable early arriving delegates to spend an evening together with peers. During the two days, the conference hosted over 60 attending delegates and provided 32 delegates with the opportunity to present their work in front of peers and experts in the field of media, communication and cultural studies. In addition to the presentations of early career researchers, the conference hosted five keynote speakers: Dr. Clare Birchall, Prof. Lisa Blackman, Dr. Rebecca Coleman, Prof. Gary Hall and Associate Professor Mandy Rose. They all spoke to the theme 'Transformative Practice and Theory: Where We Stand Today' from their field of expertise. This special issue of Networking Knowledge also includes a series of interviews with the keynote speakers where they elaborate on their work.

\section{Theme Development and Bid Application}

As three unseasoned PhD students from the Department of Media (now School of Media and Performing Arts) at Coventry University, it's safe to say we didn't quite know what we were letting ourselves in for when we first put together our bid to host the MeCCSA-PGN Conference 2015. We didn't know how much it would take over our lives, the lessons we would learn, the talented people we would meet, the connections we would forge, the impressive range of topics we would be introduced to, or the amazing level of support from staff and students alike at Coventry University that we would be lucky enough to draw on. 


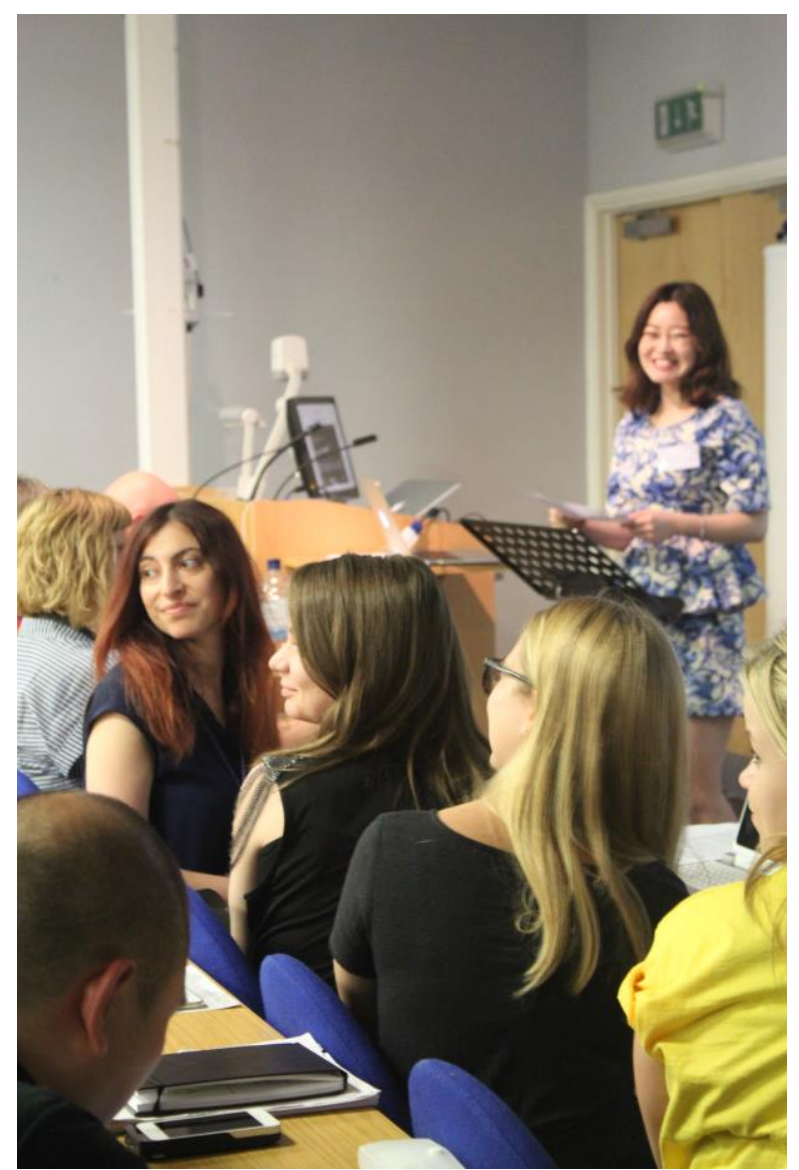

Best Paper Award ceremony (photo by D. Demicoli)

We were both apprehensive and intimidated during the process of compiling the bid and developing the theme, but by the time we had sent in our application we had already begun to feel a growing sense of excitement, and couldn't help but hope we would be given the opportunity to realise our ideas and put our plans into practice.

We wanted to take the opportunity to celebrate the very current changes in research in our fields, which varied from aspects of open and digital media, to theories such as posthumanism, and the turn to affect. We had therefore decided on the umbrella concept of 'Transformative Practice and Theory: Where We Stand Today' as our theme, and we hoped to be able to draw together a diverse range of researchers in the fields of media, communication and cultural studies. Happily, we were successful in our bid - and then the real work began! We were extremely lucky and grateful to have received such a great amount of support from Coventry University, including funding from the Centre for Disruptive Media and Coventry School of Art and Design. This enabled the conference to provide additional benefits such as Best Paper Awards, delegate bursaries, and an informal conference dinner at no added cost. Undoubtedly, the most enjoyable and exciting parts of the conference organisation were receiving fascinating abstracts in response to our call for papers. We became enthused by the prospect of hosting such insightful papers, as well as contacting experts in the fields in the hope of them speaking at our conference - and the utter joy and excitement of them saying yes! 


\section{The Keynotes}

We were delighted to have Prof. Lisa Blackman open the conference with her paper on 'Haunted Data: Social Media, Weird Science and Archives of the Future'. This timely contribution offered delegates an insight into Blackman's forthcoming book Haunted Data. The talk focused on the potential of PPPR (post-publication peer-review) and used two case studies to consider how what becomes submerged or hidden can be kept alive through traces.

Associate Prof. Mandy Rose closed day one with her highly enjoyable talk on 'Producing Participation - reflections on documentary as a co-created practice'. Sharing some of her work and the projects she had been involved in during her career, Rose spoke about forms of participation emerging within documentary and the potential of how it will develop as virtual environments and new forms of immersion come into play.

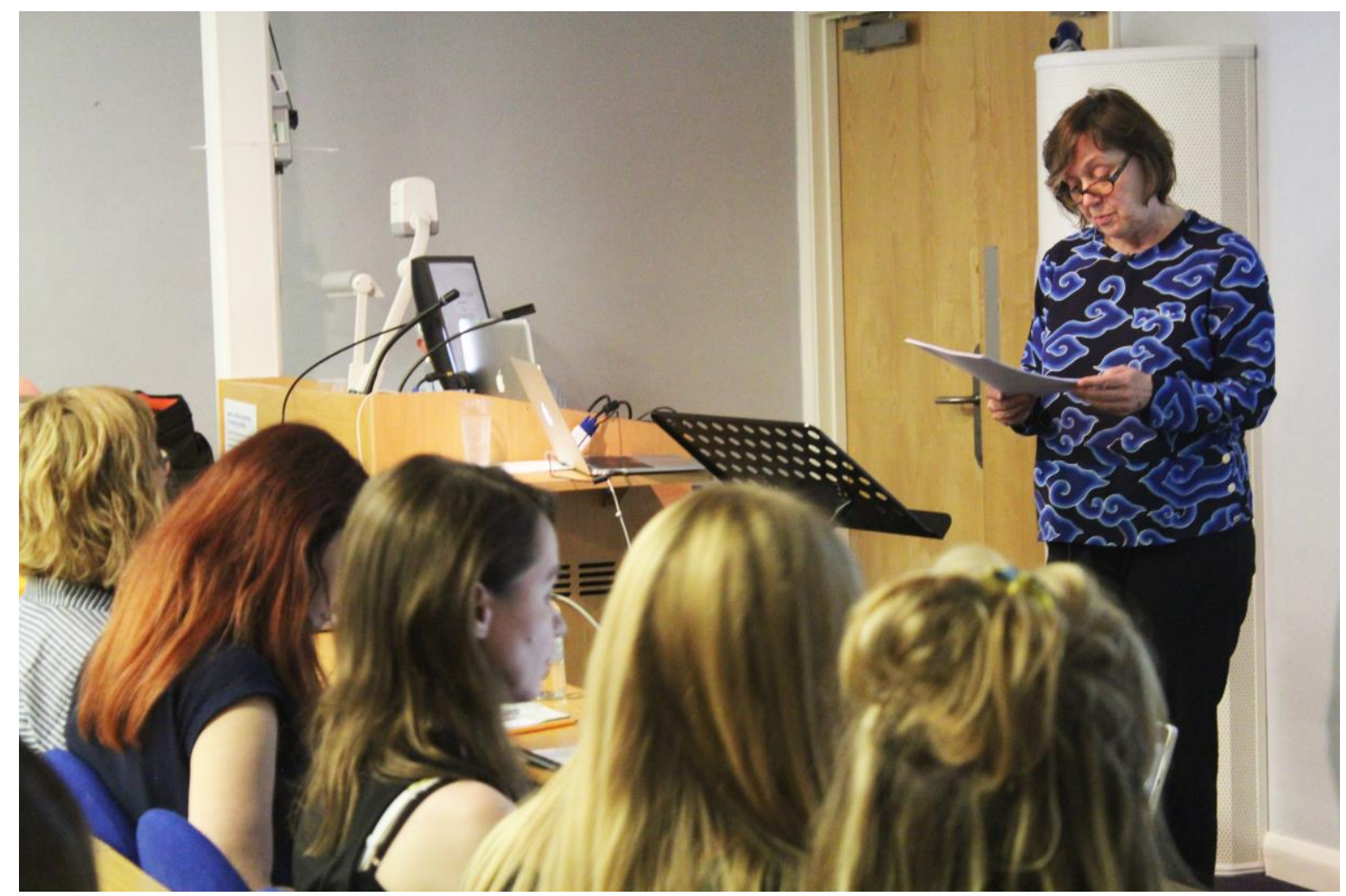

Mandy Rose's keynote presentation (photo by D. Demicoli)

The second day of the conference was opened by Dr. Rebecca Coleman, who delivered her keynote on 'Transformative Images, Temporality and Infra-Structures of Feeling'. This paper proposed a way to explore the affective relations between bodies, images and environments by developing a notion of infra-structures of feeling, and considered whether 'infra-structures of feeling' is a helpful way of thinking about the transformative potential of images.

In the closing panel Dr. Clare Birchall and Professor Gary Hall discussed 'Scholarship in the Sharing Economy'. Birchall spoke about the subjectivity arising from the sharing economy in her paper 'Shareveillance: Data, Subjectivity and Scholarship' and suggested ways of thinking more critically about what kinds of publishing, networks and communications we want to develop in the university. The closing keynote, 'How to Be Inhuman: Data Power, 
Paper Power' from Hall discussed the process of neoliberal subjectivation and considered the liberal, humanist subjectivity that we are transitioning from.

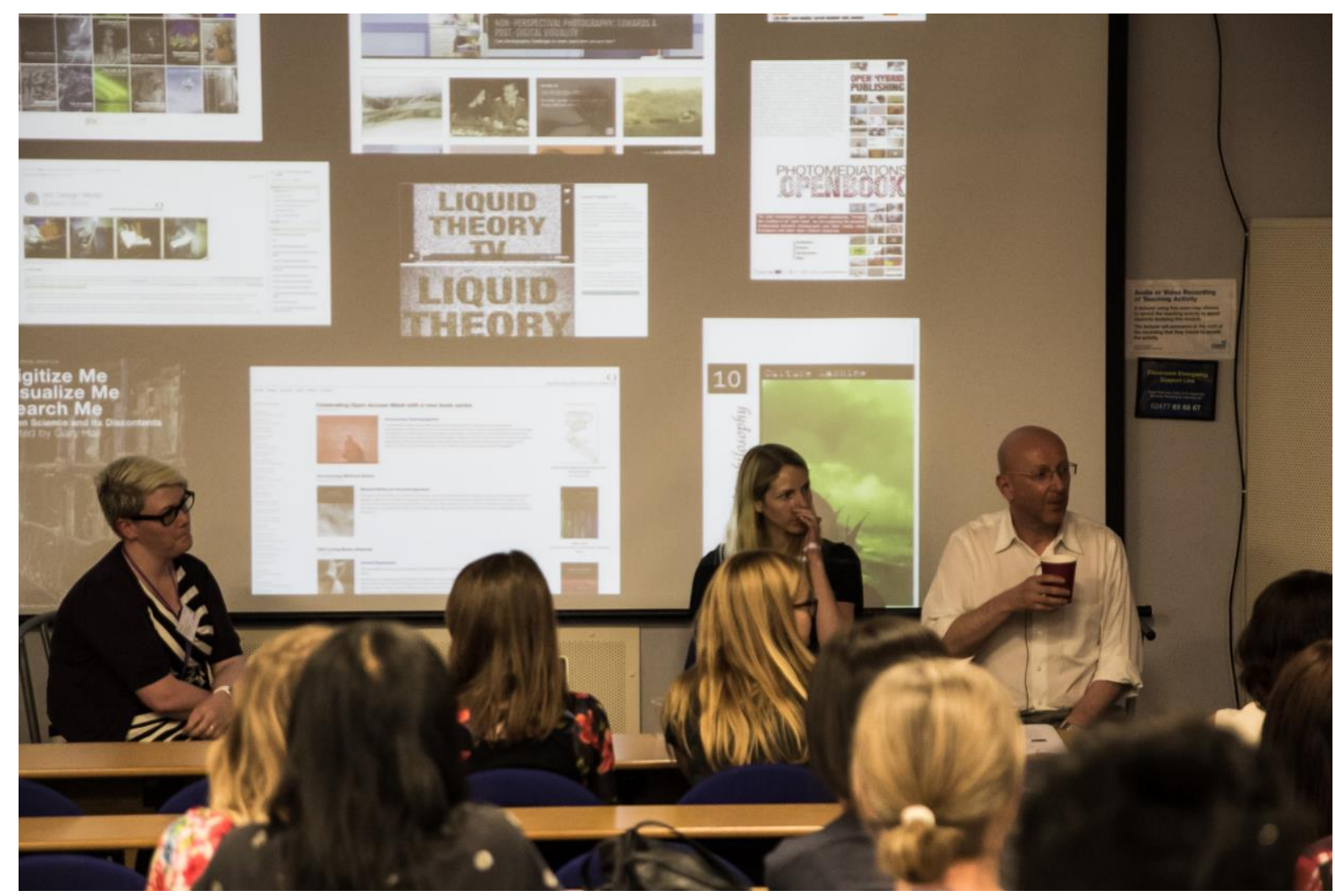

Clare Birchall and Gary Hall answering questions after their keynote (photo by A. Fareza)

These presentations were a complementary mix of innovative uses of theory and practice, and provided a thought-provoking start and end to each day, inspiring early career researchers to consider where their own research might take them in the future and how they could complicate and extend their own projects in similar ways. The interviews with the keynote speakers included in this special issue will provide readers with further insight into these topics.

\section{The Conference}

The conference took place during a few very warm summer days in July. Prepared with plenty of water and refreshments to keep the delegates hydrated, the conference took off with the screening of the documentary CitizenFour (2014). The award winning documentary fitted well with the theme, as it explored the position of Edward Snowden during the big whistleblower affair that brought attention to the critical and problematic position of privacy in contemporary computer-mediated societies. The sharing of data and information and the influence of these developments in academic practice and the formation of contemporary neoliberal subjectivities was explored in more depth by the keynote speakers, turning the screening into a fruitful and stimulating starting point for discussions amongst the delegates.

After a casual and warm start, the 'real' conference started the next day with even more sunshine and more importantly, more food for thought, discussion and inspiration. For the organising committee this was a very exciting moment that saw the culmination of months of 
work. With thoughts crossing our minds such as 'This is the right day, isn't it?!', 'We have put all the signs up, haven't we?!', 'Are you sure we have enough refreshments?!', 'Would everyone enjoy themselves?!' we spent the last few hours making sure everything was ready - but we needn't have worried. Assisted by an army of helpful, enthusiastic and hardworking student helpers and a group of skilful supportive staff members, the whole organisation of the conference went very smoothly, making sure that every delegate was looked after and providing the space for insightful and inspiring presentations throughout the two days.

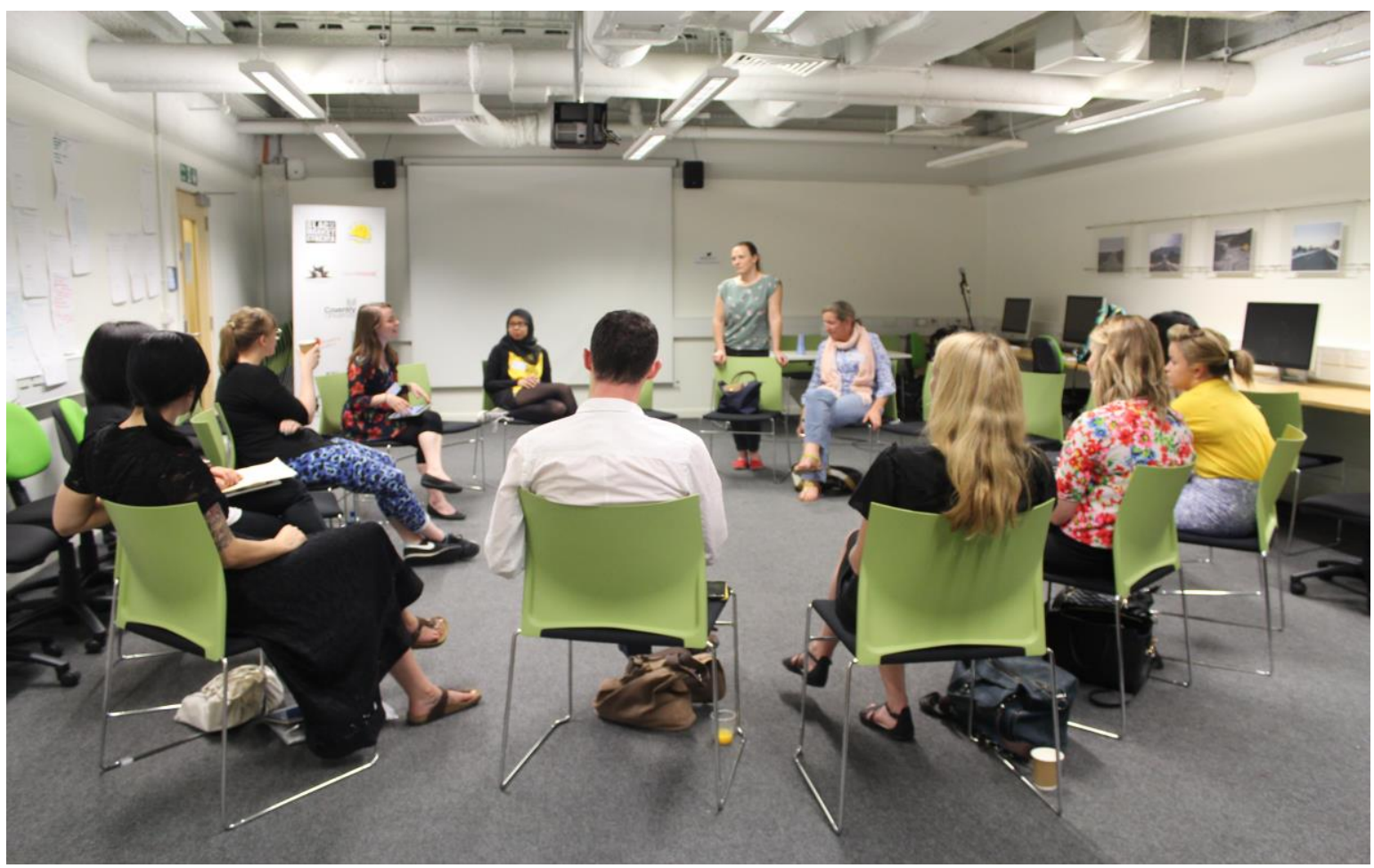

MeCCSA Women's Network workshop (photo by D. Demicoli)

The presentations were divided across eleven panels that each addressed the theme of 'Transformative Practice and Theory' from different angles. There was a fantastic variety of papers submitted for consideration for the conference, and this enabled us to create a diverse range of panels exploring contemporary questions and issues with methods/methodology, visual and sensory cultures, gaming, online identity constructions and politics of race, gender and sexuality. The MeCCSA-PGN Conference 2014, hosted by the University of Leeds, saw the introduction of the Best Paper Award, and this year we were happy to expand upon this initiative and offer two awards. The winners were Alberto Micali with his paper 'How to become war machine, or... a low hacktivist (un)methodology in pieces' and Eva Zekany with her paper 'Through the Google Glass: Configurations of attentions in the age of new media' you can find their papers in this special issue. Thanks to the global reach of the conference, the scope of the papers moved beyond the British context by exploring new and emerging subcultures across the globe e.g. Truffles Economy in Australia; Neo-Liberal Celebrities in China; Social-Economy in Nigeria; LGBT in China. Within this broad range of papers and their far reaching scope it became apparent that digital, social media and questions about data formed the basis of contemporary research practices amongst the presenting delegates. This interest was also mirrored by the three workshops that were hosted on the second day of the conference: 'Academic (De)Rigour: Blogging as Transformative Practice' from Matthew Hawkins; 'Negotiating Gender in Academia' from the MeCCSA Women's Network, 
facilitated by Francien Broekhuizen and Dr. Adrienne Evans; and 'Social Media in Egypt: Four Years after the Revolution' from Noha Atef. The workshops provided delegates with the space to interact and engage more actively with the ideas that were presented throughout the two days.

\section{Social Programme}

We understand the importance of forging connections and friendships and sharing work, and therefore ensured that, as well as providing the pre-conference film screening, the conference fee included an informal conference dinner at the end of the first day of the conference. And who can say 'no' to a night filled with free food and drinks, good company and great networking opportunities?! These ingredients made the conference dinner a great success that drew (almost) all delegates and keynote speakers to the local bar and restaurant. For those who still had some energy left, there was a great night out in a bar in an old church that was bombed in the second world war, leaving only the spire intact. This historical site hosts a lovely pub that proved to be a great location for some late night drinks, snacks and talks.

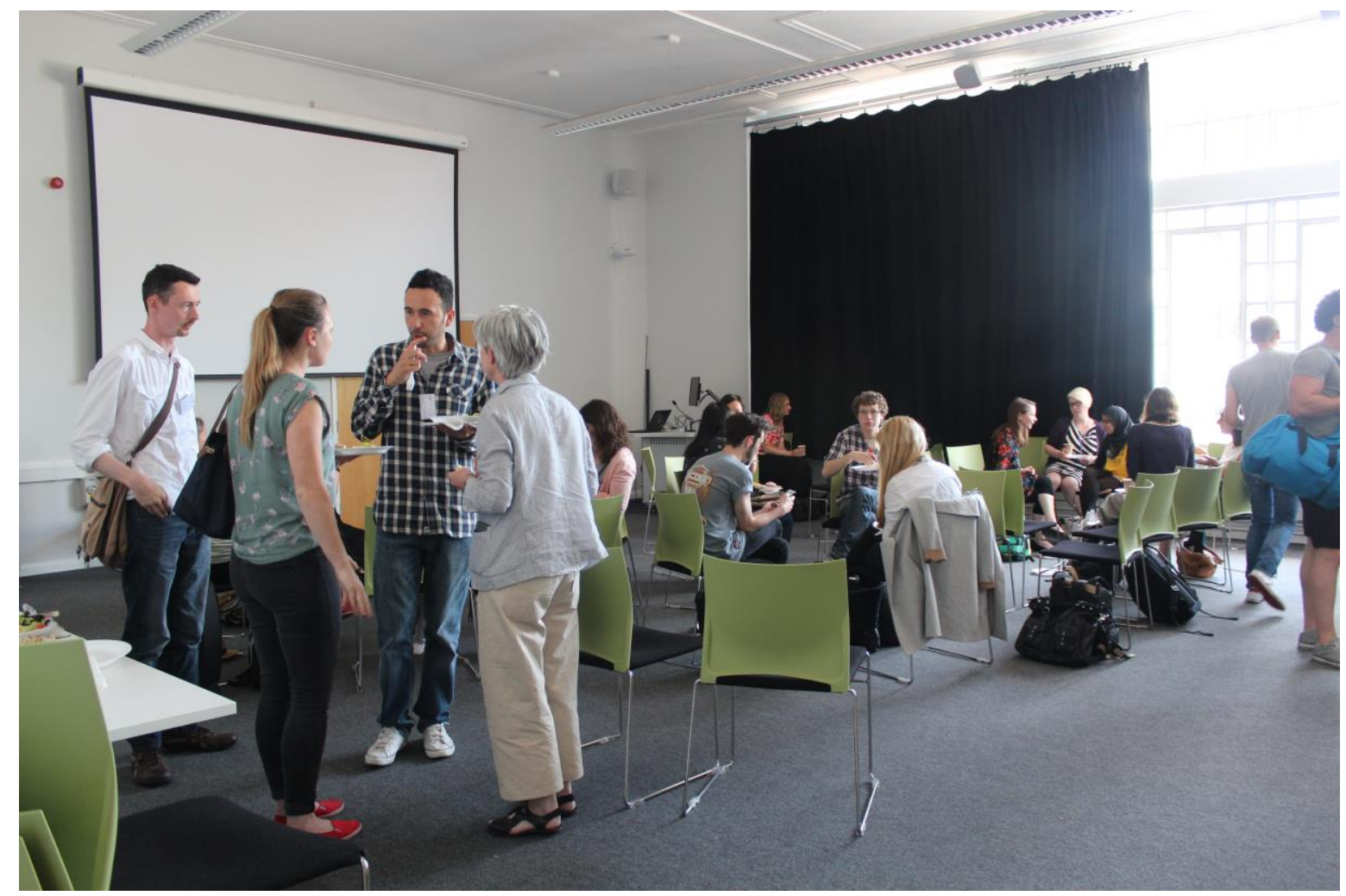

Conference delegates networking during lunch break (photo by D. Demicoli)

\section{Social Media}

Throughout the conference delegates made great use of the Twitter hashtag, which allowed delegates to engage in discussion around the specific panel they chose to go to, but also to get an idea of the other presentations, discussions and questions that were posed in other panels occurring at the same time. Tweets from the conference have been collected to create a "Storify" from each day, providing an overview of the discussions and presentations that took 
place and the delegates' experiences of coming to Coventry. The resulting digital archive of the conference also includes video of highlights of the conference and the presentations of the keynote speakers, which are all broadcast via our YouTube channel.

The incorporation of (social) media has been one of the characteristics of the conference, addressing our theme and question of 'Where Do We Stand Today'. During the organisation, we asked ourselves the question of how media can be used to cater the running up to the conference, the conference itself, and the aftermath. In doing so we aimed to move away from the old-fashion canvas bags, extensive paper programmes, leaflets etc. Instead we utilised an interactive scheduling platform from Sched.org that allowed delegates to create their own profile, interact with other delegates and explore the programme throughout the conference on their mobile devices. The programme was also show-cased on different screens throughout the conference venue, enabling delegates to stay up to date with the programme and their choices of where to go and what to do next. This app and our own website still host biographical details from all presenters at the conference as well as all abstracts in full. The design of the conference poster and website was assigned to a recent graduate of the Photography course at Coventry University, Joseph Kesisoglou, who enthusiastically took up this opportunity to enrich his portfolio.

In addition to our website, Sched programme, YouTube channel, Google+ page, Facebook event, Twitter feed and Storify creations we're delighted to have been involved in the process of co-editing this special issue of Networking Knowledge as a lasting testament to the varied works presented at the MeCCSA-PGN Conference 2015. We'd like to extend our thanks to Simon Dawes, the journal editor, for including us in this process, and to all those delegates of the conference who submitted their papers for consideration. One of the main things we will take away from our MeCCSA-PGN Conference 2015 experience is inspiration from the high quality of research taking place amongst up-and-coming academics. We're proud to be involved in this issue amongst our peers, and look forward with anticipation to watching the careers of those around us develop and grow as they continue to transform theory and practice through their (and hopefully our!) work.

Francien Broekhuizen is a PhD student at Coventry University. Her research focuses on the interactions between digital and off-line cultures. In doing so she has a particular interest in the constructions of different temporalities, bodies and matter. Her recent work, 'Pain, Pleasure and Bridal Beauty', is published in the Journal of Gender Studies and focuses on the way brides negotiate their bridal bodily becoming in a post-feminist sentiment.

Email: broekhuf@uni.coventry.ac.uk

Danai Mikelli is a PhD Candidate in the School of Media and Performing Arts at Coventry University. Her doctoral research provides an ethnographic exploration of a series of interventions introducing interactive documentary in the context of Critical Media Education. She was a member of the 2015 MeCCSA PGN Conference organising committee.

Email: mikellid@uni.coventry.ac.uk 
Poppy Wilde is a PhD student in the School of Media and Performing Arts at Coventry University and was co-organiser of the MeCCSA PGN Conference 2015. Her background is in performance studies and drama, and her autoethnographic $\mathrm{PhD}$ project explores the lived experience of MMORPG gaming with particular focus on the gamer as one embodiment of posthuman subjectivity. Her research interests are posthumanism, digital cultures, embodiment, performance in online contexts and the lived experience in research methods.

Email: wildep@uni.coventry.ac.uk 